\title{
Characterizing the transition dynamics for multi-pulsing in mode-locked lasers
}

\author{
Brandon G. Bale ${ }^{a}$, Khanh Kieu ${ }^{b}$, Frank Wise ${ }^{b}$ and J. Nathan Kutz ${ }^{c}$ \\ ${ }^{a}$ Photonics Research Group, Aston University, Birmingham UK B4 7ET; \\ ${ }^{a}$ Department of Applied Physics, Cornell University, Ithaca, NY 14853; \\ ${ }^{c}$ Department of Applied Mathematics, University of Washington, Seattle, W A 98195-2420
}

\begin{abstract}
We consider experimentally and theoretically a refined parameter space near the transition to multi-pulse modelocking. Near the transition, the onset of instability is initiated by a Hopf (periodic) bifurcation. As cavity energy is increased, the band of unstable, oscillatory modes generates a chaotic behavior between single- and multi-pulse operation. Both theory and experiment are in good qualitative agreement and they suggest that the phenomenon is of a universal nature in mode-locked lasers at the onset of multi-pulsing from $N$ to $N+1$ pulses per round trip. This is the first theoretical and experimental characterization of the transition behavior, made possible by a highly refined tuning of the gain pump level.
\end{abstract}

Keywords: mode-locked lasers, multi-pulsing, harmonic mode-locking

\section{INTRODUCTION}

The onset of multi-pulsing as a function of increasing laser cavity energy is a ubiquitous phenomenon observed in mode-locking. ${ }^{1,2}$ Indeed, the multi-pulsing dynamics has been demonstrated in a wide variety of theoretical and experimental configurations with both passive and active laser cavities ${ }^{3}-{ }^{16}$ Until recently, there was no satisfactory theoretical understanding of the instability mechanism that initiated the multi-pulsing process. However, theoretical progress on a mode-locked laser cavity based upon a waveguide array (WGA) architecture led to a significant theoretical advancement in quantifying the multi-pulsing process. ${ }^{17}$ Indeed, the analysis associated with the multi-pulsing instability showed that the underlying transition from $N$ to $N+1$ pulses per round trip occurred in a nontrivial manner. Specifically, a linear stability analysis of the mode-locked solution shows that, as the gain increases, the stable pulse solution undergoes an oscillatory Hopf bifurcation which leads to a stable mode-locked breather solution. Increasing the gain further leads to a transition from the breather to a two pulse per round trip steady-state configuration. The transition process is chaotic in nature, with the solution dynamically switching back and forth between the breather and the two-pulse state over a range of gain parameters, before the two pulse per round trip state stabilizes at higher gain. However, irrespective of the saturable absorption mechanism used for mode-locking, i.e. a passive polarizer, carbon nanotube, wave-guide array, quantum well saturable absorber, etc., this transition phenomenon seems to be fairly universal in modelocking models. In this manuscript, a highly-sensitive experimental study is performed near the multi-pulsing instability threshold in a laser mode-locked with single-walled carbon nanotube (SWCNT) saturable absorption. The experimental findings confirm qualitatively the theoretical multi-pulsing predictions ${ }^{17}$ and suggests that the phenomenon is relevant to a broad range of mode-locked laser cavity configurations, regardless of the specific saturable absorption mechanism. To our knowledge, this is the first experimental study of its kind and shows the key role that the gain saturation plays in determining the nonlinear, dynamical transition in mode-locked laser cavities.

Theoretical progress on the multi-pulsing transition has often been hampered by the theoretical difficulty in characterizing the instabilities of mode-locked solutions in various laser cavity configurations. ${ }^{2}$ Indeed, a full analytic treatment of the characteristic instabilities of mode-locked solutions in the master mode-locking equation has only recently been given. ${ }^{18,19}$ Much of this difficulty is due to the fact that the gain saturation

Further author information: (Send correspondence to J. N. Kutz)

J. N. Kutz: E-mail: kutz@amath.washington.edu, Telephone: 12066853029

Fiber Lasers VII: Technology, Systems, and Applications, edited by Kanishka Tankala, Jay W. Dawson, Proc. of SPIE Vol. 7580, 75801Z · @ 2010 SPIE · CCC code: 0277-786X/10/\$18 - doi: 10.1117/12.840543 


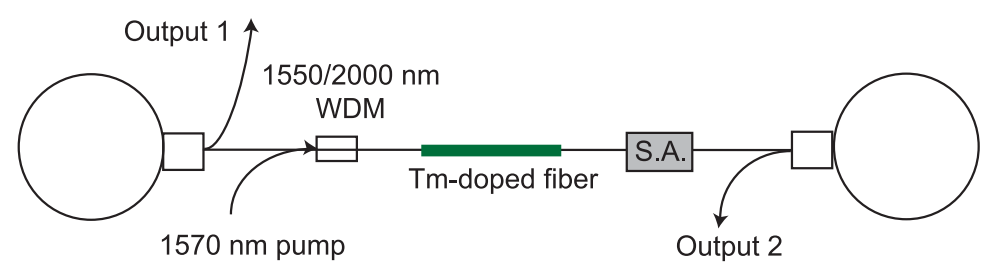

Figure 1. Schematic of the experimental setup. The saturable absorber (S.A.) used is single-walled carbon nanotubes.

acts as a nonlocal term in the governing equations, making a standard linear stability analysis highly nontrivial. And yet, the gain saturation term is critical in determining the overall stability of the system. Ignoring the term, or treating it as a constant, completely misses the multi-pulsing stability transition and leads to erroneous conclusions about the stability of the mode-locked pulses in general. Here, for mathematical convenience only, we consider the saturable absorption mechanism in the laser cavity to be generated by a waveguide array. The primary advantage of the waveguide array based laser cavity ${ }^{17,20,21}$ is that exact solutions can be calculated and their stability explicitly analyzed, and as the gain is increased, robust multi-pulse operation occurs. This is unlike the qualitative master mode-locking equation which is not very robust and suffers from collapse and blowup of the mode-locked solution near the multi-pulsing transition point. But it should be emphasized that the waveguide array is not the mechanism responsible for generating the chaotic behavior, is is simply the saturable absorption mechanism for initiating the mode-locking process. The mode-locked solutions are first found to undergo a Hopf bifurcation to stable, periodic breathers as the gain is increased in the cavity. The resulting periodic breathers are then destabilized and a chaotic behavior is observed as the cavity energy intermitingly switches between one and two pulses. A slight increase in gain brings the pulses finally into the stable multi-pulsing state for which the inter-pulse dynamics becomes important. ${ }^{3,15,22}$ The range of gain values for which this oscillatory and chaotic transition happens is quite small, suggesting that it could be easily missed in experiment if a refined study is not performed near the transition point. Further, this process repeats itself when transitioning from $N$ to $N+1$ pulses per round trip. ${ }^{17}$ Our analysis showed that harmonic mode-locking can be predicted by the gain model

$$
\left.g_{(} Z\right)=\frac{2 g_{0}}{1+N\left\|A_{0}\right\|^{2} / e_{0}},
$$

which gives the gain parameters for which $N$ pulses per round trip configurations exist, assuming that $g_{0}$ corresponds to a stable one-pulse solution. The theory also predicts bi-stability between several stable $N$ pulse per round trip solutions, which is also in qualitatively agreement with experimental findings.

The paper is outlined as follows: In Sec. 2, a brief overview is given of the experimental configuration used to generate mode-locked pulses. Section 3 outlines the basic theoretical model used for predicting the transition phenomena at the multi-pulsing threshold. Section 4 highlights the computational and experimental findings and shows the qualitative agreement between theory and experiment in regards to the periodic and chaotic transition regions. A brief overview of the results and its implications are given in Section 5.

\section{EXPERIMENTAL SETUP}

Although the transition from single to multi-pulse operation is a ubiquitous phenomena in pulsed laser systems, here we investigate this phenomena using a soliton thulium-doped fiber laser ${ }^{23}$ shown in Fig. 1. It contains $\sim 70$ $\mathrm{cm}$ of Tm-Ho co-doped fiber with a $9 \mu \mathrm{m}$ core diameter and $\sim 60 \mathrm{~dB} / \mathrm{m}$ absorption at $1570 \mathrm{~nm}$. The cavity is formed between two loop mirrors (with reflectivities $\sim 40$ and 5 percent) based on $2 \times 2$ fused couplers. It should be noted that the fiber ends at the outputs have to be angle cleaved to avoid detrimental feedback. The saturable absorber used are SWCNTs, which are fabricated using the technique reported previously. ${ }^{24}$ In this laser, the SWCNTs have an outer diameter $\sim 1.5 \mathrm{~nm}$ so as to place the absorption band near $2 \mu \mathrm{m}$. The total dispersion of the laser cavity is estimated to be $\sim-0.2 \mathrm{ps}^{2}$.

Mode-locking occurs when the pump power is increased to $320 \mathrm{~mW}$, however the laser tends to start in a multiple-pulsing regime. Single pulse operation is achieved by reducing the pump power to $\sim 200 \mathrm{~mW}$. The laser 

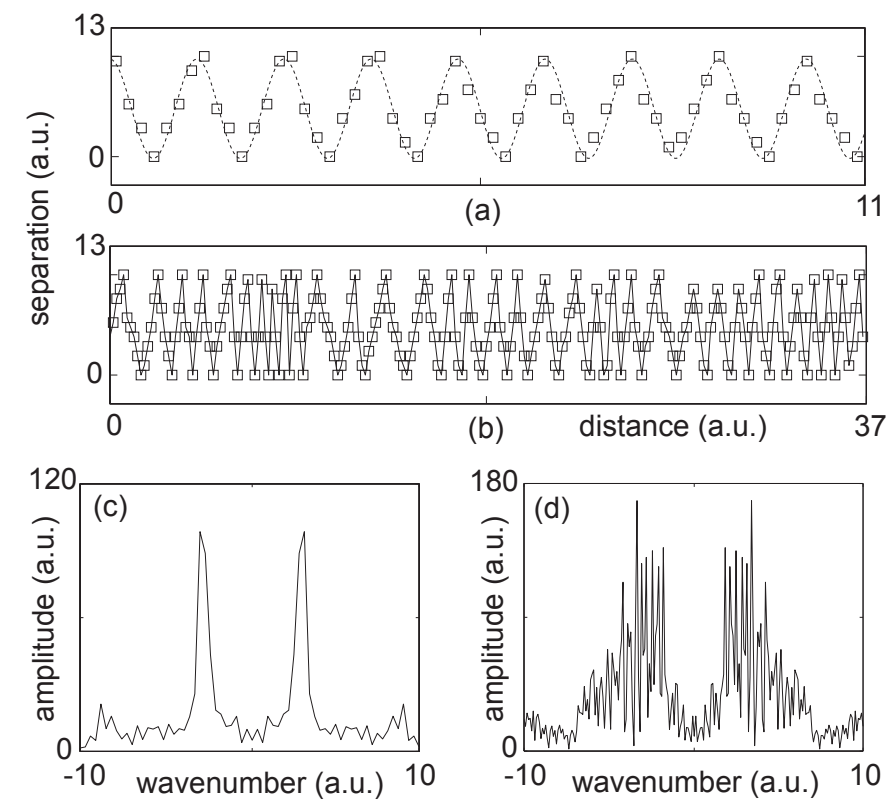

Figure 2. Experimentally measured time series of the separation of two pulses once single pulse operation transitions to multi-pulsing. (a) For a certain pump power, the separation of the two pulses is nearly periodic. A best fit sinusoidal function (dashed) is fit to the data (squares) and shows excellent agreement. (b) For increasing pump power, the separation of the two pulses is not periodic, and evolves irregularly. The Fourier transform of the time series is shown in (c) for the periodic case and (d) for the irregular case. Note that the spectrum in (d) is a signature that the separation dynamics is chaotic.

is quit stable and produces a pulse with duration of $750 \mathrm{fs}$ and a $9 \mathrm{~nm}$ bandwidth. From this pump level the gain was increased in small intervals from $200 \mathrm{~mW}$.

As the pump level is increased from $200 \mathrm{~mW}$, the single pulse undergoes an instability and transitions into a breather. Further increasing the pump level leads to a transition regime in which the cavity energy oscillates between single and two-pulse operation. To characterize the dynamics, we measure the pulse separation of the "two pulses" (when separation is zero, it is a single pulse). Figure 2(a) shows the time series of the separation as the pump level is increased. The Fourier transform of the time series highlights that two dominant modes exist (Fig. 2(c)), typical of periodic behavior. Increasing the pump level, the oscillation period becomes more irregular as seen in the time series in Fig. 2(b). Taking the Fourier transform shows that indeed there are more than two modes present in the oscillations (Fig. 2(d)). Further, the dense, rapidly varying modal distribution shown here is characteristic of chaotic behavior.

In general, the transition from one- to two-pulse operation consists of an interesting regime where the pulse is in-between the two states. Within this region, as the pump level is increased the oscillations between the two states becomes more irregular, until finally stable two-pulse operation is obtained. Although we have focused on the one to two pulse transition, we have experimentally observed the same dynamics between any $N$ to $N+1$ transition.

\section{GOVERNING EQUATIONS}

There are a large number of theoretical models ${ }^{2}$ that have been developed for quantifying the energy equilibration and mode-locking exhibited by numerous experimental configurations. ${ }^{1}$ To make progress on quantifying the transition dynamics observed in a mode-locked laser cavity, we consider a specific theoretical model where the intensity discrimination is provided by the nonlinear mode-coupling in waveguide arrays. ${ }^{17,20,21}$ We emphasize that this is for convenience only in illustrating the transition phenomena. Indeed, the experimental configuration considered is mode-locked not by waveguide arrays, but by the saturable absorption action of the SWCNTs 


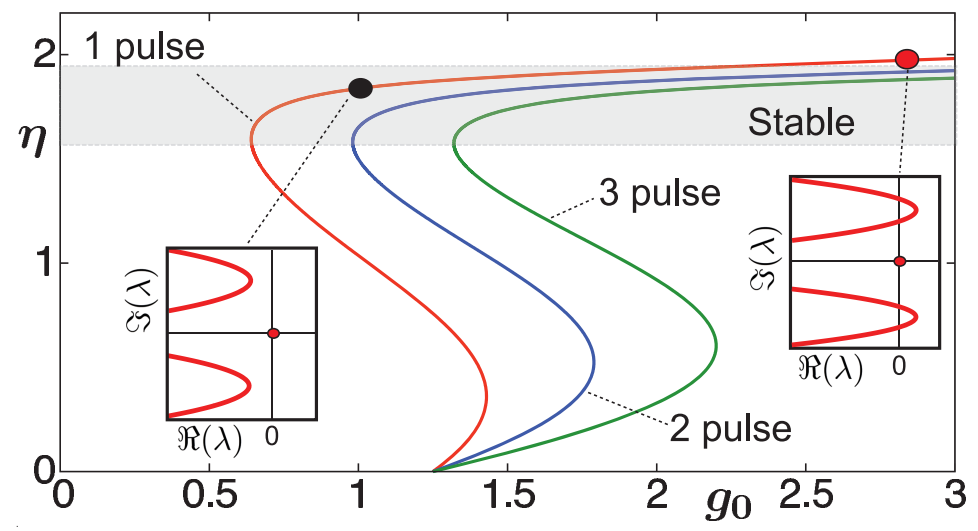

Figure 3. Mode-locked solution branches of the form (4) with (5). Depicted are the 1-pulse branch $(N=1)$, 2-pulse branch $(N=2)$ and 3-pulse branch $(N=3)$ along with their corresponding stability region (shaded gray region). The inset demonstrates the linear (spectral) instability that occurs near the Hopf bifurcation point of the 1-pulse branch of solutions. As the gain increases past the Hopf bifurcation point, bands of oscillatory, unstable modes cross into the right half plane leading to a breather solution and chaotic dynamics.

described in the previous section. Regardless, the transition phenomenon considered holds in both cavities and exhibits the universal nature of the multi-pulsing transition behavior.

When placed within an optical fiber cavity, the pulse shaping mechanism of a waveguide array leads to stable and robust mode-locking. ${ }^{17,20,21}$ In its most simple form, the nonlinear mode-coupling is averaged into the laser cavity dynamics ${ }^{17}$ to generate a master equation for the WGA-driven mode-locking dynamics. Symmetry considerations in the WGA and computational studies of the mode-locking dynamics predicts that the evolution dynamics in the WGA mode-locked cavity can be accurately represented by ${ }^{17,20}$

$$
\begin{aligned}
& i \frac{\partial u}{\partial z}+\frac{1}{2} \frac{\partial^{2} u}{\partial t^{2}}+\gamma|u|^{2} u+C v+i \delta_{0} u-i g(z)\left(1+\tau \frac{\partial^{2}}{\partial t^{2}}\right) u=0 \\
& i \frac{\partial v}{\partial z}+C(w+u)+i \delta_{1} v=0 \\
& i \frac{\partial w}{\partial z}+C v+i \delta_{2} w=0
\end{aligned}
$$

where the saturated gain (energy equilibration) behavior ${ }^{1}$ is given by

$$
g(z)=\frac{2 g_{0}}{1+\|u\|^{2} / e_{0}} .
$$

with $\|u\|=\int_{-\infty}^{\infty}|u|^{2} d t$. Here the $u(z, t), v(z, t)$ and $w(z, t)$ represent the normalized electromagnetic fields in the center waveguide $(u)$ and two neighboring waveguides $(v$ and $w)$. The variables $z$ and $t$ present the normalized propagation distance and time respectively. Note that the equations governing the neighboring WGA fields are ordinary differential equations. All fiber propagation and gain effects occur in the central waveguide $(u)$ since this is the only waveguide that experiences saturated gain. This model accounts for all the leading-order physical effects in the cavity including the chromatic dispersion in the central waveguide, self-phase modulation $(\gamma)$, linear attenuation in the three waveguides $\left(\delta_{0}, \delta_{1}\right.$ and $\delta_{2}$ respectively), WGA evanescent linear coupling $(C)$, linear gain $\left(g_{0}\right)$, bandwidth-limit on the gain $(\tau)$, and energy saturation $\left(e_{0}\right)$. A detailed analysis of the scalings can be found in Refs. ${ }^{17,20}$ Note that we are only considering the anomalous dispersion laser cavity in order to be consistent with the experimental findings. However, multi-pulsing is also observed in normal dispersion laser cavities as well.

It is this approximate system which will be the basis for our analytic findings and transition phenomenon studies. The primary reason for considering this model is the the fact that Eq. (2) provides a great deal of 
analytic insight due to its hyperbolic secant solutions

$$
u(z, t)=\eta \operatorname{sech} \omega t^{1+i A} e^{i \theta z},
$$

where the solution amplitude $\eta$, width $\omega$, chirp parameter $A$, and phase $\theta$ satisfy a set of nonlinear equations. ${ }^{17}$ Note that although this chirped pulse solution is the same as that found in the master mode-locking equation, ${ }^{1}$ the mode-locked solution here is a global attractor, is not susceptible to blow-up and is highly robust under perturbation. Indeed, stability of this solution, its Hopf bifurcation, chaotic transition, and corresponding solutions with $N$ pulses per round trip can all be explored in a semi-analytic fashion. ${ }^{17}$

To understand the stability of the multi-pulsing solutions, once can consider a laser cavity with $N$ modelocked pulses of the form (4). Assuming the pulses are well-separated so that any pulse-to-pulse overlap can be neglected, the multi-pulse solution can be constructed by assuming a solution of the form (4) with the gain (3) modified to be ${ }^{17}$

$$
g(z)=\frac{2 g_{0}}{1+N\|u\|^{2} / e_{0}} .
$$

The simple step of including the number of pulses $N$ in the saturating gain gives the $N$ pulse mode-locked state. Figure 3 demonstrates the stability and solution branches for $N=1,2$ and 3 . The gray region in this figure denotes the area of stable mode-locked solutions. Stability is determined by linearizing about the steady-state solution (4) and considering the associated eigenvalue problem. Linear instability occurs for any eigenvalue whose $\Re\{\lambda\}>0 .{ }^{17}$ The insets on the 1-pulse solution branch $(N=1)$ illustrate the Hopf instability mechanism computed previously. ${ }^{17}$ Note that there is an entire band of unstable modes if one proceeds far enough beyond the Hopf bifurcation point. It is conjectured that this large number of unstable modes is ultimately responsible for the chaotic behavior observed in the multi-pulsing transition. Linear stability no longer holds in this case since the solutions are now time-periodic and full Floquet-type analysis is required. This analysis shows that the model (2) provides an ideal analytic framework and model for characterizing the multi-pulse transition.

\section{MULTI-PULSE TRANSITION: THEORY AND EXPERIMENT}

To make a qualitative comparison with the experimental findings of Sec. 2, simulations are performed and evaluated near the theoretically predicted Hopf transition point of (2). Unlike the previously analysis of Kutz and Sandstede, ${ }^{17}$ emphasis is placed on the pulse-to-pulse interaction of the transition to demonstrate and confirm the experimental findings. Although the focus here will be on the transition from one to two pulses, a similar transition is predicted to occur from $N$ to $N+1$ pulses. ${ }^{17}$

Figure 4 demonstrates the mode-locking dynamics as a function of the gain strength parameter $g_{0}$. Over the range of values $g_{0}=2.3,2.52,2.53,2.68,2.72$ and 2.75, the mode-locking is observed to go from 1-pulse per round trip to 2-pulses per round trip. During the transition, the Hopf (periodic) bifurcation is clearly observed (top right panel of Fig. 4) to preceed the onset of chaotic dynamics. Specifically, the breather solution begins to breath erratically and drift while intermittingly forming two pulses and then one again. For sufficiently high gain, two stable mode-locked pulses are formed. To make connection with experimental findings of this process, the distance between neighboring pulses is computed for the simulations shown in Fig. 4 with $g_{0}=2.68$ and $g_{0}=2.72$. For these two gain values, Fig. 5 shows the pulse-to-pulse distance and its Fourier transform with the DC component removed. The oscillations for these two gain values corroborate the experimental findings by showing a dominant periodic signature $\left(g_{0}=2.68\right)$ and a chaotic signature $\left(g_{0}=2.72\right)$. Thus the multi-pulsing transition behavior is qualitatively consistent in both theory and experiment. A quantitative comparison is not possible due to aliasing in the experimental video footage as well as the fact that the theoretically based WGA model is different from the SWCNT mode-locking of the experiment. Specifically, in the experimental movies in which each frame was on the order of a fraction of a second, several million round trips of the cavity will have elapsed. Thus the visualization misses many of the faster timescale events that are captured in the simulations. Thus when comparing, it should be noted that the separation between the pulses of simulation are not expected to match the experimental video. At best, we can investigate the Fourier spectra in order to ascertain the periodic or chaotic signatures. Regardless, both models exhibit the key features predicted, suggesting the broader nature of the phenomena. ${ }^{17}$ 

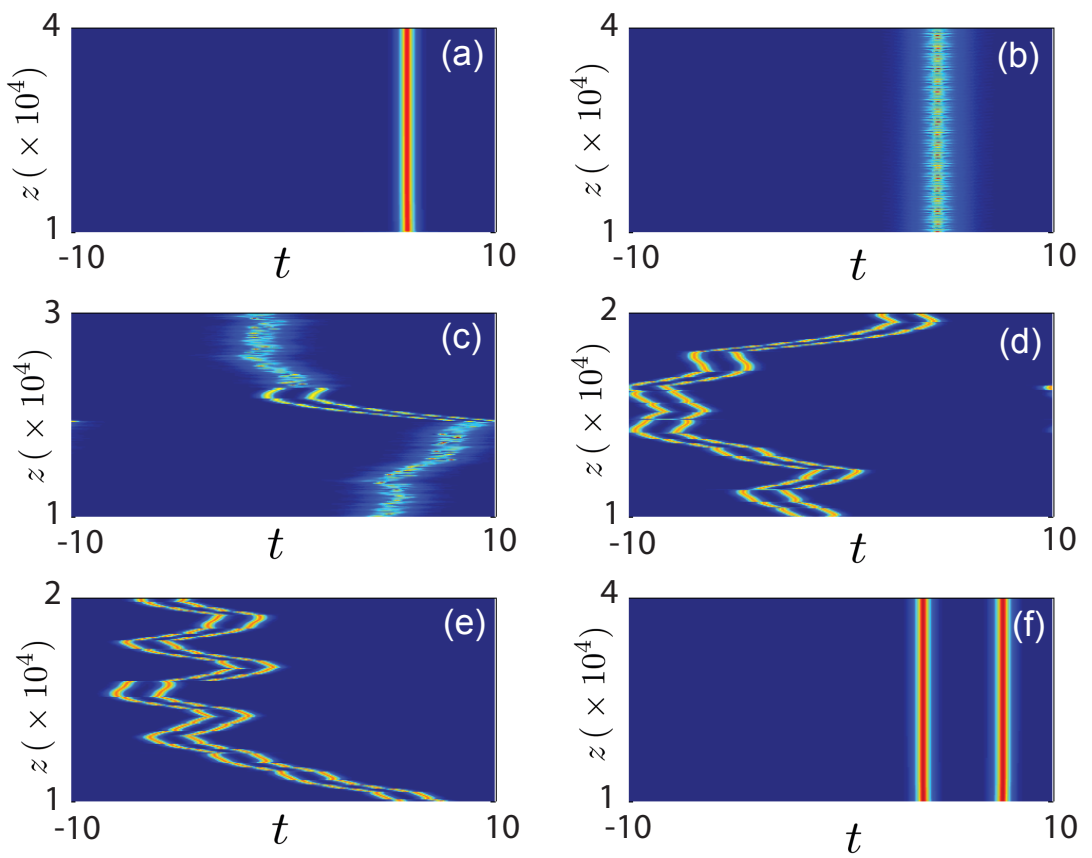

Figure 4. Mode-locking dynamics as a function of increasing gain pumping $g_{0}=2.3$ (a), 2.52 (b), 2.53 (c), 2.68 (d), 2.72 (e) and 2.75 (f). As the gain is increased, mode-locking is observed to go from 1-pulse per round trip to 2-pulses per round trip via a Hopf bifurcation followed by a chaotic regime of interaction. The parameters for numerical simulation are $\gamma=8, C=5, \delta_{0}=\delta_{1}=0, \delta_{2}=10, \tau=0.1$ and $e_{0}=1 .^{17}$

\section{CONCLUSION}

The phenomenon of multi-pulsing in mode-locked lasers has been well-known for almost two decades, with its energy quantization and pulse-to-pulse interactions having been studied and quantified by numerous researchers ${ }^{3}-$ .${ }^{16}$ Indeed, there are still many open problems and competing theories concerning the fundamental physical mechanisms that drive the multi-pulse interaction. Despite the various studies, this manuscript provides for the first time, to our knowledge, a detailed study of the transition dynamics when the cavity bifurcates form $N$ to $N+1$ pulses per round strip. As is clearly evident in the theory and experiment, periodic and chaotic behavior occurs very close to the transition point of the instability. The initial instability is shown to be driven by a Hopf bifurcation as a band of unstable eigenvalues cross into the right half plane, i.e. the real part of the eigenvalues are positive, leading to growth and instability. It is conjectured that as the gain is increased further the large number of oscillatory modes that cross into the right-half plane generate a complicated oscillatory (chaotic) structure, thus leading to the observation of seemingly random oscillations in the pulse-to-pulse interactions. A further increase in gain leads to stable $N+1$ mode-locking once again.

Although the theory and experiments are in qualitative agreement, there remain open questions about the pulse-to-pulse interactions. Specifically, the role of the translationally invariant zero mode in forcing the translation of the pulse as observed in the panels of Fig. 4. Additionally, what the phase dynamics and how do they drive the pulse-to-pulse separation. And finally, what determines the length scale of separation for the mode-locked pulses once stabilized in the $N+1$ configuration.

\section{Acknowledgements}

J. N. Kutz acknowledges support from the National Science Foundation (DMS-0604700) and the Air Force Office of Scientific Research (FA9550-09-0147). 

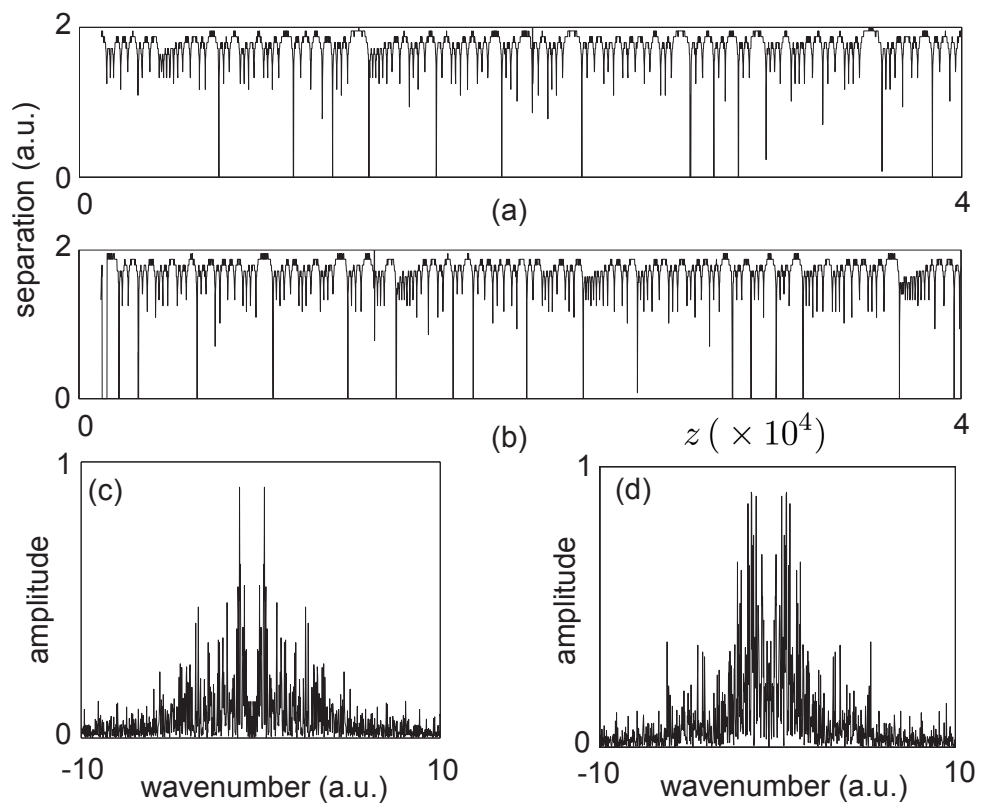

Figure 5. Numerical simulations of the pulse-to-pulse distance and its Fourier transform for two different gain values. For the top panel, $g_{0}=2.68$ and a strong periodic signature is observed in the spectrum in (c). As the gain is increased to $g_{0}=2.72$ in (b), the periodicity is lost and a stronger chaotic motion is observed as illustrated in the spreading of the spreading of the spectral signatures in (d). Note that the DC signature has been zeroed out in the spectrum. Aside from $g_{0}$, the parameters are the same as those used in Fig. 4.

\section{REFERENCES}

[1] H. A. Haus, "Mode-Locking of Lasers," IEEE J. Sel. Top. Quantum Electron. 6, 1173-1185 (2000).

[2] J. N. Kutz, "Mode-locked Soliton Lasers, SIAM Review 48, 629-678 (2006).

[3] J. N. Kutz, B. C. Collings, K. Bergman, and W. H. Knox, "Stabilized Pulse Spacing in Soliton Lasers Due to Gain Depletion and Recovery", IEEE J. Quant. Elec., 34, 1749-1757 (1998).

[4] B. Collings, K. Berman, and W. H. Knox, "Stable multigigahertz pulse train formation in a short cavity passively harmonic modelocked Er/Yb fiber laser", Opt. Lett., 23, 123-125 (1998).

[5] M. E. Fermann and J. D. Minelly, "Cladding-pumped passive harmonically mode-locked fiber laser", Opt. Lett., 21, 970-972 (1996).

[6] A. B. Grudinin, D. J. Richardson, and D. N. Payne, "Energy quantization in figure eight fibre laser", Electron. Lett., 28, 1391-1393 (1992).

[7] M. J. Guy, P. U. Noske, A. Boskovic, and J. R. Taylor, "Femtosecond soliton generation in a praseodymium fluoride fiber laser", Opt. Lett., 19, 828-830 (1994).

[8] M. Horowitz, C. R. Menyuk, T. F. Carruthers, and I. N. Duling III, "Theoretical and experimental study of harmonically modelocked fiber lasers for optical communication systems," J. Lightwave Tech. 18, 1565-1574 (2000).

[9] R. P. Davey, N. Langford, and A. I. Ferguson, "Interacting solutions in erbium fibre laser", Elec. Lett., 27, 1257-1259 (1991).

[10] M.J. Lederer, B. Luther-Davis, H.H. Tan, C. Jagadish, N.N. Akhmediev, and J.M. Soto-Crespo, "Multipulse operation of a Ti:Sapphire laser mode locked by an ion-implanted semiconductor saturable-absorber mirror", J. Opt. Soc. Am. B, 16, 895-904 (1999).

[11] Q. Xing, L. Chai, W. Zhang, and Ch.-yue Wang, "Regular, period-doubling, quasi-periodic, and chaotic behavior in a self-mode-locked Ti:sapphire laser", Opt. Comm., 162, 71-74 (1999).

[12] M. Lai, J. Nicholson, and W. Rudolph, "Multiple pulse operation of a femtosecond Ti:sapphire laser", Opt. Comm., 142, 45-49 (1997). 
[13] Ch.-yue Wang, W. Zhang, K.F. Lee, and K.M. Yoo, "Pulse splitting in a self-mode-locking Ti:sapphire laser", Opt. Comm., 137, 89-92 (1997).

[14] H. Kitano and S. Kinoshita, "Stable multipulse generation from a self-mode-locked Ti:sapphire laser", Opt. Comm., 157, 128-134 (1998).

[15] A. N. Pilipetskii, E. A. Golovchenck, and C. R. Menyuk, "Acoustic effect in passively mode-locked fiber ring lasers", Opt. Lett., 20, 907-909 (1995).

[16] S. Namiki, E.P. Ippen, H. Haus, and C.X. Yu, "Energy equations for mode-locked lasers", J. Opt. Soc. Am. B, 14, 2099-2111 (1997).

[17] J. N. Kutz and B. Sandstede, "Theory of passive harmonic mode-locking using wave-guide arrays," Opt. Express 16, 636-650 (2008).

[18] T. Kapitula, J. N. Kutz, and B. Sandstede, "Stability of Pulses in the Master-Modelocking Equation", J. Opt. Soc. Am. B 19, 740-746 (2002).

[19] T. Kapitula, J. N. Kutz, and B. Sandstede, "The Evans function for nonlocal equations," Indiana J. Math. 53, 1095-1126 (2004).

[20] B. G. Bale, J. N. Kutz and B. Sandstede, "Optimizing waveguide array mode-locking for high-power fiber lasers," IEEE J. Sel. Top. Quantum Electron. 15 220-231 (2009).

[21] J. Proctor and J. N. Kutz, "Theory and Simulation of Passive Mode-Locking with Waveguide Arrays," Opt. Lett. 13, 2013-2015 (2005).

[22] J. P. Gordon, "Interaction forces among solitons in optical fibers," Opt. Lett., 8, 396-398 (1983).

[23] K. Kieu and F. Wise, "Soliton Thulium-Doped Fiber Laser with Carbon Nanotube Saturable Absorber", IEEE Phot. Tech. Lett., 21, 128-130 (2009).

[24] K. Kieu and M. Mansuripur, "Femtosecond laser pulse generation with a fiber taper embedded in carbon nanotube/polymer composite," Opt. Lett., 32, 2242-2244 (2007). 